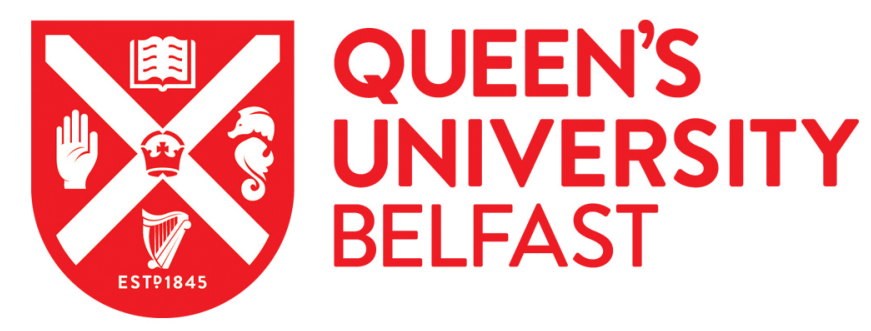

\title{
Place versus response learning in fish: a comparison between species.
}

McAroe, C. L., Craig, C. M., \& Holland, R. A. (2015). Place versus response learning in fish: a comparison between species. Animal Cognition, 1-9. https://doi.org/10.1007/s10071-015-0922-9

\section{Published in:}

Animal Cognition

\section{Document Version:}

Peer reviewed version

Queen's University Belfast - Research Portal:

Link to publication record in Queen's University Belfast Research Portal

Publisher rights

http://link.springer.com/article/10.1007\%2Fs10071-015-0922-9

\section{General rights}

Copyright for the publications made accessible via the Queen's University Belfast Research Portal is retained by the author(s) and / or other copyright owners and it is a condition of accessing these publications that users recognise and abide by the legal requirements associated with these rights.

Take down policy

The Research Portal is Queen's institutional repository that provides access to Queen's research output. Every effort has been made to ensure that content in the Research Portal does not infringe any person's rights, or applicable UK laws. If you discover content in the Research Portal that you believe breaches copyright or violates any law, please contact openaccess@qub.ac.uk. 
1 Place versus Response Learning in Fish: A Comparison between

2 Species

3 Claire L. McAroe ${ }^{1,2}$ cmcaroe01@qub.ac.uk, +44 (0) 2892664256

4 Cathy M Craig ${ }^{2}$ and Richard A Holland ${ }^{1}$

$5{ }^{1}$ School of Biological Sciences, Queen's University Belfast, Medical Biology

6 Centre, 97 Lisburn Road, Belfast, BT9 7 BL, Northern Ireland

$7 \quad{ }^{2}$ School of Psychology, Queen's University Belfast, University Road, Belfast,

$8 \quad$ BT7 1NN, Northern Ireland

9

10

11

12

13

14

15

16

17

18

19

20

21

22

23

24

25 
27 Place learning is thought to be an adaptive and flexible facet of navigation.

28 Due to the flexibility of this learning, it is thought to be more complex than the

29 simpler strategies such as learning a particular route or navigating through the 30 use of cues. Place learning is crucial in a familiar environment as it allows an

31 individual to successfully navigate to the same endpoint, regardless of where 32 in the environment the journey begins. Much of the research to date focusing 33 on different strategies employed for navigation has used human subjects or 34 other mammals such as rodents. In this series of experiments, the spatial 35 memory of four different species of fish (goldfish, killifish, zebrafish and 36 Siamese fighting fish) was analysed using a plus maze set-up. Results suggest that three of the species showed a significant preference for the adoption of a place strategy during this task whereas zebrafish showed no significant preference. Furthermore, zebrafish took significantly longer to learn the task than the other species. Finally, results suggest that zebrafish took the

41 least amount of time (seconds) to complete trials both during training and 42 probe.

43 Keywords: Spatial memory, Spatial cognition, Fish, Animal model, Navigation

\section{Introduction}

45 In the animal kingdom, spatial memory is important for many reasons

46 including avoiding predation, prey detection and finding a mate (Wolbers \&

47 Hegarty 2010; White \& Brown 2014). There are two categories associated

48 with spatial memory and navigation (van Gerven et al. 2012). The first are

49 called "egocentric processes" and involve encoding features of the

50 environment in relation to where the individual is currently located (Shelton \& 
51 McNamara 2001). These processes involve learning to navigate to a location using landmarks in the environment as beacons or by learning a series of

53 turning responses (O'Keefe \& Nadel 1978; van Gerven et al. 2012).

54 Egocentric navigation is relatively simple and therefore allows spatial

55 memories to be formed easily (van Gerven et al. 2012). Such methods do,

56 however, lack flexibility, particularly in response to changes within the

57 environment, meaning the individual will be less likely to reach their desired

58 location from a novel start point (Rodriguez et al. 1994).

59 O'Keefe and Nadel argued that animals are also able to navigate using

60 another set of processes which, although more cognitively demanding, are

61 able to adapt more readily to changes within the environment (O'Keefe \&

62 Nadel 1978; Rodriguez et al. 1994). These are called "allocentric processes".

63 Cognitive mapping, first proposed by Tolman, is the best known example of

64 an allocentric process and is described as a complex mental representation of

65 a familiar environment that can be used to influence spatial behaviour and

66 decision-making (Tolman 1948; O’Keefe \& Nadel 1978). One of the key

67 features of such an allocentric process is that an individual is able to navigate

68 based on the spatial relationships between multiple landmarks, sensory

69 features and possible routes within a particular environment (laria et al. 2009).

70 As the individual moves though the environment, its cognitive map is

71 continuously updated, making for more robust spatial memory that is also

72 more relevant and current (Cheeseman et al. 2014). This means that changes

73 within the environment, such as removal of specific landmarks or attempting

74 to reach the same goal location from a novel start point, will not significantly

75 affect the individual's spatial memory (Wolbers \& Hegarty 2010). 
Many experiments have been conducted using maze tests to analyse spatial memory in a variety of species, with mammals and birds being the most popular animals to study (Tolman et al. 1946; O'Keefe \& Nadel 1979; Clayton \& Krebs 1995; Shettleworth \& Westwood 2002; Hamilton et al. 2009). Both taxa possess a hippocampal structure in the brain which is thought to be

81 a key area associated with complex spatial memory abilities (see: Pravosudov \& Roth II 2013). Some studies have also shown that individuals who navigate through complex environments on a regular basis, or perform complex spatial memory tasks, are likely to have an enlarged hippocampus (Clayton \& Krebs 1995; Maguire et al. 2000). However, the lack of a hippocampal structure in other animal classes does not necessarily mean that such species are incapable of flexible navigation.

Fish are the most numerous of all vertebrate species (Casebolt et al. 1998) and many live in complex environments, some of which are subject to instability and therefore require the animals to have powerful spatial memory capabilities (Brown 2014). Rodriguez and colleagues showed that, like mammals and birds, goldfish are capable of navigating using both egocentric and allocentric processes during maze tests (Rodriguez et al. 1994). The same authors have also been able to manipulate navigational strategy by ablating brain regions of fish, directly suggesting that there are specific areas of the fish brain responsible for different navigational processes (Salas et al. 1996; López et al. 2000; Broglio et al. 2010). Despite these suggestions, a recent search on Web of Knowledge (January 2015) using "spatial memory" as the search criterion reported 54,109 articles with only 216 citing fish as the 100 study species. Furthermore, many of these articles focus on the large-scale 
101 navigation abilities (i.e. migration) of particular species rather than on which

102 strategies fish use to solve spatial tasks (Dittman \& Quinn 1996; Saito \&

103 Watanabe 2005). Finally, there have been limited studies comparing the

104 similarities and differences in spatial memory across species of fish with many

105 experiments focusing on only one study species (e.g. Braithwaite \& De Perera

106 2006; Shapiro \& Jensen 2009; Lamb et al. 2012). Such research would

107 highlight that complex spatial memory is an attribute of fish as a taxonomic

108 group rather than just of specific species.

109 This study used four different species of fish to assess the length of

110 time each took to learn a spatial task. It also analysed which strategy each

111 species preferred (egocentric or allocentric) for spatial memory. The four

112 species were goldfish, Siamese fighting fish, zebrafish and a type of killifish.

113 Each of these species is teleost (from the class Actinopterygii) with three from

114 the order Cypriniformes and Siamese fighting fish from the order Perciformes

115 (www.fishbase.org). It is important that they are all from the same class, as

116 the teleost brain is thought to be very similar across fish species, and is

117 comparable on some levels to the mammalian brain (Tropepe \& Sive 2003).

118 As mentioned previously, the hippocampus is thought to be crucial for

119 allocentric spatial memory performance in mammals, the area associated with

120 similar spatial memory abilities in teleost fish has been identified as the lateral

121 pallium area of the telencephalon, and impairment to this area results in a

122 marked reduction in allocentric spatial learning in these animals (see: Broglio

123 et al. 2003 for a review).

124 All but one of these species (killifish) are thought of as domesticated

125 fish (Gordon \& Axelrod 1968; Andrews 2002; Spence et al. 2011) and popular 
126 laboratory species in the studies of a variety of both physiological and

127 cognitive experiments (Roitblat et al. 1982; Kishi 2004; Portavella \& Vargas

128 2005). The final species was chosen as it is gaining popularity within similar

129 fields (Herrera \& Jagadeeswaran 2004). Goldfish are the most domesticated

130 of all, and therefore there is limited available information about their natural

131 habitat. However as descendants of carp, it is assumed they tend to occupy

132 ponds and other such bodies of water (Andrews 2002). More is known about

133 the natural habitats of the other three species, each of which also prefer still

134 or slow-moving pools of water (Gordon \& Axelrod 1968; Genade et al. 2005;

135 Spence et al. 2011). This is important in a comparison, as a previous study

136 has indicated that even within the same species, fish from different

137 environments (fast flowing vs. still) may use different strategies for navigation

138 (Odling-Smee \& Braithwaite 2003). Finally, the species of fish range in

139 sociality with zebrafish having an innate tendency to shoal (Spence et al.

140 2011), through to the killifish and Siamese fighting fish where it is

141 recommended that the males are housed separately.

142 Materials and Methods

143 Subjects

144 Twenty fish from the following four species were used, giving a total of 80

145 individuals; Carassius auratus auratus (common goldfish), Nothobranchius

146 guentheri (killifish), Betta speldens (Siamese fighting fish), and Danio rerio

147 (zebrafish). The mean length of each species (with standard deviation) was

$1487.63 \pm 2.47 \mathrm{~cm}, 3.58 \pm 1.08 \mathrm{~cm}, 8.63 \pm 1.12 \mathrm{~cm}$, and $4.14 \pm 0.88 \mathrm{~cm}$, respectively. All

149 Siamese fighting fish were male, six of the killifish were male and fourteen

150 female, sex of goldfish and zebrafish was unknown. All animals were 
151 commercially sourced (goldfish, Siamese fighting fish and zebrafish from

152 Exotic Aquatics, Belfast, N. Ireland; killifish from Maidenhead Aquatics,

153 Newtownabbey, N. Ireland). All animals were experimentally naïve.

\section{Housing Conditions}

155 All apparatus was commercially sourced (Maidenhead Aquatics,

156 Newtownabbey, N. Ireland and Exotic Aquatics, Belfast, N. Ireland). Goldfish

157 were kept in $80 \mathrm{~cm} \times 30 \mathrm{~cm} \times 40 \mathrm{~cm}$ tanks with a stocking density of five in

158 each. Temperatures were maintained at an average of $20^{\circ} \mathrm{C}$. During

159 experimentation, killifish, Siamese fighting fish and zebrafish were housed

160 individually (for identification purposes) at an average temperature of $25^{\circ} \mathrm{C}$.

161 Individual housing jars were made of transparent glass and were kept beside

162 each other allowing fish to have visual access to conspecifics. When not

163 completing experiments, all fish were fed commercial flaked food. $\mathrm{pH}$ and

164 waste levels in all tanks were monitored regularly using API Freshwater

165 Master Test Kit and water changes were carried out on a regular basis. Waste

166 levels were kept within safe ranges (0ppm ammonia \& nitrite; <40ppm nitrate).

$167 \mathrm{pH}$ range for goldfish was maintained at a range of $7.6 \pm 0.2 ; 7.7 \pm 0.3$ for

168 killifish and zebrafish; and $8.1 \pm 0.1$ for Siamese fighting fish. All fish were

169 maintained in a 13: $11 \mathrm{~h}$ light: dark cycle at all times during the laboratory.

170 Experimental Design

171 Apparatus 
Perspex blocking arm during training - this arm would become start arm for probe trials

Fig. 1 Layout of experimental T-maze formed by blocking arm directly opposite start arm with a piece of Perspex. Reverse layout would be used as the T-maze for probe trials

Start arm - this arm would be blocked during probe trials

A square tank measuring $63 \mathrm{~cm} \times 63 \mathrm{~cm} \times 43 \mathrm{~cm}$ was used for all trials. Water temperatures were similar to housing conditions for each species and waste levels were kept within the same safe limits. $\mathrm{pH}$ was maintained at a range of $7.8 \pm 0.4$ (depending on species). A plus maze (similar to that used in OdlingSmee \& Braithwaite 2003) was made using pieces of white opaque Perspex, $21 \mathrm{~cm}$ in length with each arm attached to the inner walls of the tank (see Fig. $1)$.

\section{Experimental Design}

Fish were placed into the arm of the maze designated as the "start arm" (see

Fig 1). Both the start arm and the arm opposite were blocked with removable pieces of Perspex. Trials would begin by unblocking the start arm to allow fish access to the remaining two arms of the maze (a T would be formed by keeping the opposite arm blocked; see Fig 1). All trials (training and probe) were timed using a standard stopwatch and recorded using a Sony HDRCX190E handycam video camera. The position of the camera and that of the experimenter was varied during experiments to help eliminate any unwanted cues. Similarly, the source of illumination was not in the vicinity of the experimental tank.

\section{Training Trials}


194 Fish were randomly assigned to receive a bloodworm reward in the arm either 195 to the left or to the right of the start arm ( $\mathrm{n}=10$ for each side within each species). A trial was considered complete when the tail fin of the fish had

197 passed fully into either arm of the maze. If the fish swam to its assigned

198 rewarded arm, it would receive bloodworm immediately and be moved back to 199 the start arm for the next trial. If the fish swam to the unrewarded arm, exit 200 from that arm would be blocked and the fish would receive a two-minute "time 201 out" (no reward given) before being moved back to the start arm for the next 202 trial. Bloodworm was the recommended food reward for each of the four 203 species by the commercial suppliers and has been used for feeding and 204 reward in a variety of experiments involving fish (e.g. Miklósi \& Andrew 1999; 205 Saito \& Watanabe 2005; Thomas et al. 2008; Pike et al. 2010). Each fish 206 would receive 10 trials per day (one block). Training criterion was a minimum 207 of 8 out of 10 trials correct for three consecutive blocks. For each incorrect 208 block (7 or fewer trials correct), the training was reset to Block One. The 209 water, along with any substrate, in the tank was disturbed between each trial 210 and the tank filtered for a minimum of 20 minutes between each fish to help

211 reduce the risk of olfactory cues. Potential cues within the maze were 212 controlled where possible, e.g. by mirroring the layout so that the external 213 filter tube and the heater (both turned off during experiments) were at 214 opposing corners of the tank and a tube was placed at the opposite wall of the 215 tank from the output tube of the filter. Outside the maze, there was a wall at 216 the end of the left hand arm while there was no wall at the end of the right 217 arm. Potential global cues (i.e. those external to the maze) were not controlled 218 in order to allow fish to avail of cues external to the tank. Such cues consisted 
219 of features on the surrounding walls (such as paper record sheets) and

220 housing tanks.

221 Probe Trial

222 When a fish had reached training criteria, it was immediately transferred to the 223 previously blocked arm, which would now become the start arm; the training

224 start arm would now be blocked to form a "T". The probe trial would begin and 225 end in the same manner as in training trials. During the probe trial, all fish 226 were rewarded regardless of choice of arm. If a fish swam to the same 227 location as before, a "place-strategy" was recorded; if they chose to swim 228 using the same turning-direction as they were trained, a "response-strategy" 229 was recorded. Following the probe trial, each fish was returned to its housing 230 tank and experimentation for that individual animal was complete.

231 Statistical Analyses

232 Data were analysed with the SPSS statistical package (20.0 version) and 233 Microsoft Excel 2010. Generalized linear models were used to assess the 234 effect of species on number of blocks to reach training criteria (Poisson 235 loglinear Model) and to assess the effect of species on time taken to complete 236 the training and probe trials (linear scale response). Pairwise comparisons 237 (Fisher's LSD method) were performed to analyse differences between 238 species following each generalized linear model. Paired-sample t-tests were 239 used to assess the difference in time taken to complete the first day versus 240 last day of training within each species. Individual binomial tests and Bayesian

241 inferences were carried out to assess whether the number of fish within each 242 species adopting a place versus response strategy differed from chance.

\section{Ethical note}


244 No invasive procedures were performed and no animals were harmed. Fish

245 numbers were the minimum required for sufficient data collection and

246 analyses. Strict procedures were followed in accordance with the 'Guidelines

247 for the treatment of animals in behavioural research and teaching' (2012).

248 Complete water changes were avoided as these can be harmful and stressful

249 to animals (see above for more information regarding water changes).

250 Furthermore, fish were always transferred in water via containers. Laboratory

251 conditions were inspected by the Veterinary Services Division of the

252 DHSSPS, Northern Ireland who deemed no licence was required for this

253 series of experiments.

\section{Results}

255 Acquisition Time

256 Acquisition time was based on the number of blocks it took each fish to reach

257 training criteria.

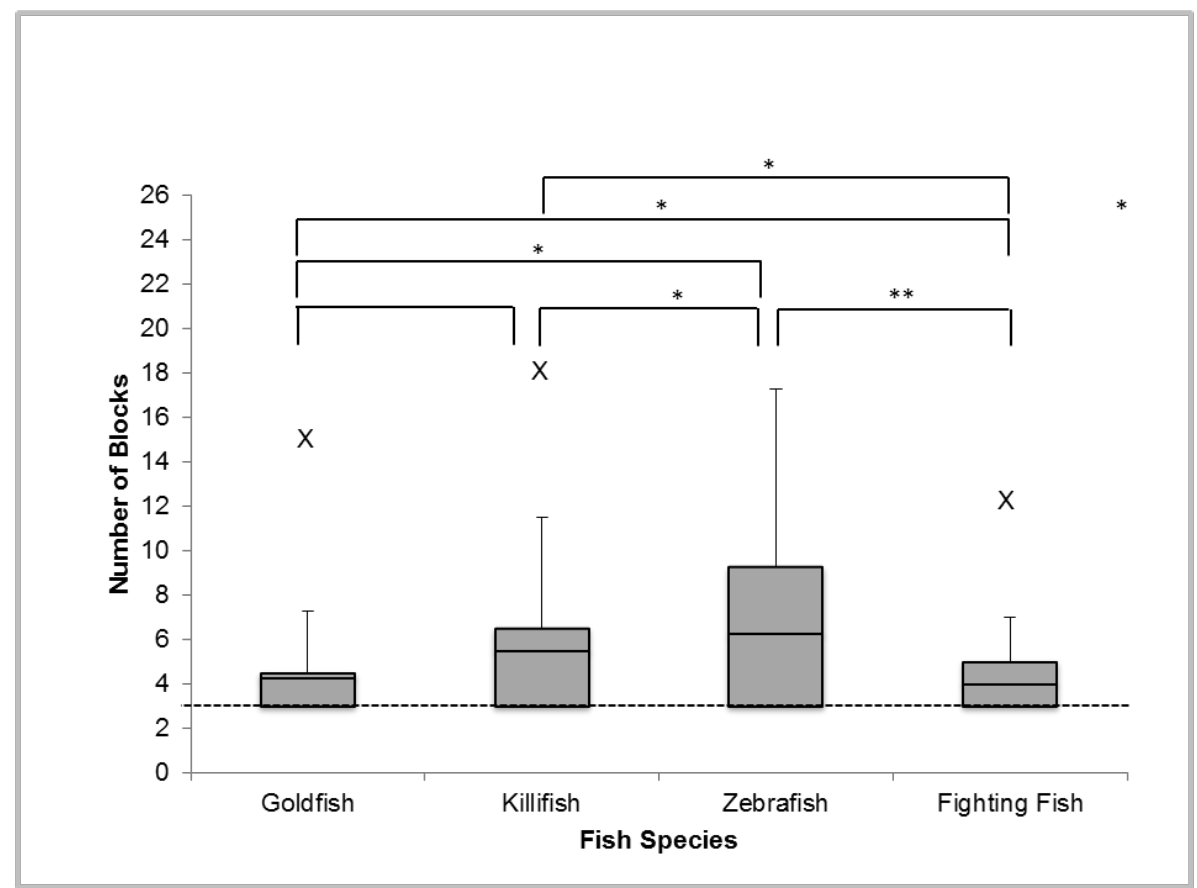


range marked with an $\mathrm{X}$ on the plot. The dotted line displays the minimum number of blocks to reach training criteria within each species ( 3 blocks in all species). On the graphical display, asterisks are used to show significant differences between species (a single asterisk $\left({ }^{*}\right)$ indicates $P \leq .05$ and double asterisks ( $\left.{ }^{* *}\right)$ indicate $P<.001$ )

265 There was a significant main effect of species on task acquisition time: Wald $X^{2}(d f=3)=25.59 ; P<0.001$. Further analyses suggest that zebrafish required significantly more blocks to learn the task than the other three species (goldfish, $P=.003$; killifish, $P=.02$; Siamese fighting fish $P<.001$ ) and that

Siamese fighting fish also took significantly fewer blocks than both goldfish $(P$ $=.05)$ and killifish $(P=.013)$. There was no significant difference between

271 goldfish and killifish $(P=.57)$ (see Fig. 2).

272

$\underline{\text { Time Taken during Training Trials }}$

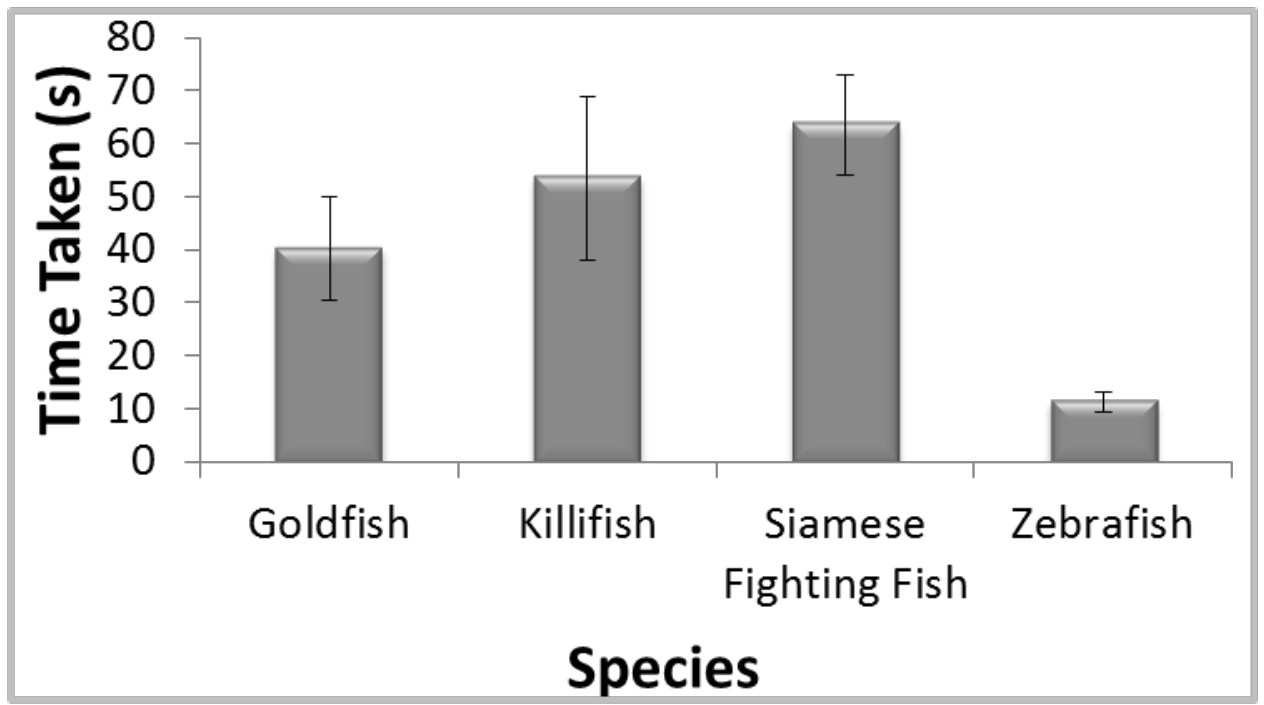

Fig. 3 Bar chart showing the mean time taken in seconds by each species to complete the training trials. Error bars indicate the standard error of the mean time taken for individual fish within each species

278 There was a significant main effect of species on the time taken (in seconds)

279 to complete individual training trials (both correct and incorrect): Wald

$280 \quad X^{2}(d f=3)=303.52 ; P<.001$. Posthoc analyses showed that zebrafish took

281 significantly less time to complete trials than the other three species $(P<.001$

282 in all cases). Furthermore, goldfish took significantly less time during training 
trials than both killifish and Siamese fighting fish (both, $P<.001$ ), and killifish

284 took less time than Siamese fighting fish $(P=.006)$ (see Fig. 3).

Difference in Time Taken on First and Last Day of Training

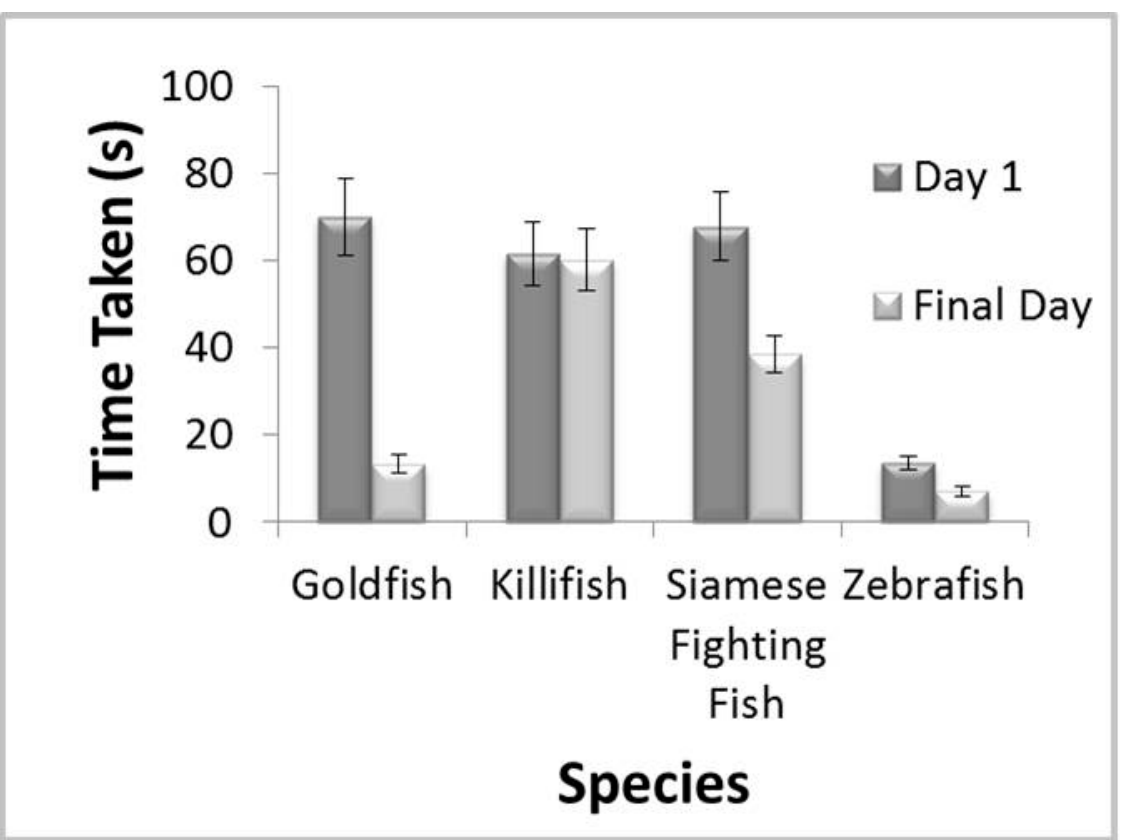

Fig. 4 Bar chart showing mean time taken in seconds by each species to complete trials on the first day of training versus on the last day of training. Error bars indicate the standard error of the mean time taken for individual fish within each species

Goldfish $(P<.001)$, Siamese fighting fish $(P=.001)$ and zebrafish $(P<.001)$

291 each took significant more time (seconds) to complete trials on the first day of

292 training compared to the last day of training. However, there was no

293 significant difference in the time taken by killifish on the first and last day of

294 training $(P=.883)$. (See Fig. 4).

295 Navigational Strategy 


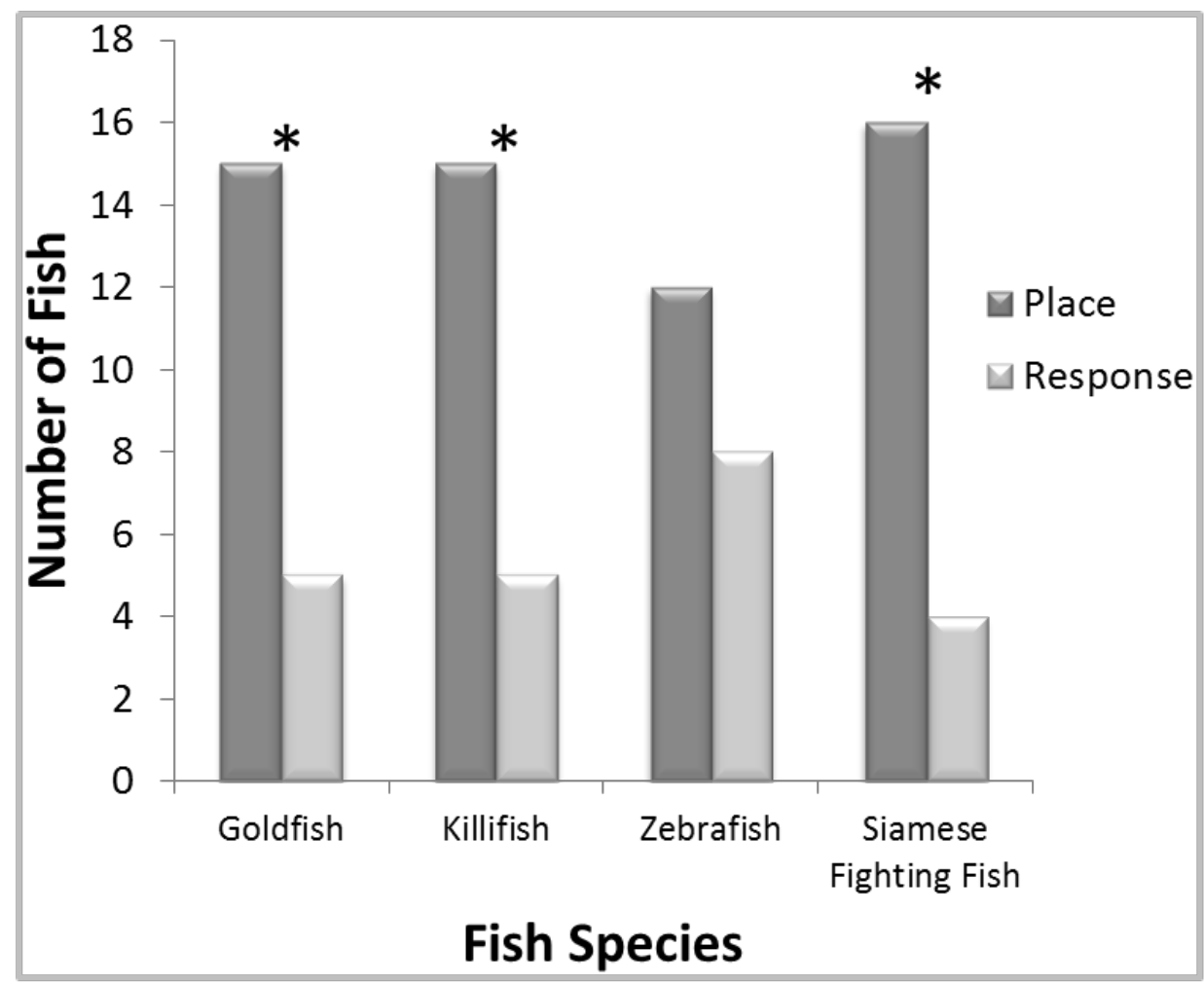

Fig. 5 Bar chart showing the number of fish in each species that adopted either a place or a response strategy during probe trials. An asterisk (*) indicates that there was a significant difference in the number of fish that adopted a place strategy over a response strategy $(P<0.5)(n$ $=20$ in all species)

301 Fig. 5 shows the number of individual fish in each species that adopted either

a place or response strategy during the probe trial.

303 Goldfish, killifish and Siamese fighting fish showed a significant preference for 304 adopting a place strategy (goldfish and Killifish, $P=0.041$; Siamese fighting 305 fish, $P=0.012)$ but zebrafish showed no significant preference $(P=0.507)$. 306 Bayesian inferences however showed no significant support for choosing the 307 null hypothesis (performance at chance level) over alternative hypothesis (of 308 place learning) for zebrafish as probability estimates of the null hypothesis lie 309 within the estimates of the alternatives with probabilities of between 0.6 and 0.8 (see Table 1). 


\begin{tabular}{cc}
\hline $\begin{array}{c}\text { Alternative } \\
\text { Hypothesis }\end{array}$ & $\begin{array}{c}\text { Evidence Ratio for } \\
\text { Null }\end{array}$ \\
\hline 0.5 (Null) & 1.00 \\
0.6 & 0.67 \\
0.7 & 1.05 \\
0.8 & 5.42 \\
\hline
\end{tabular}

Table 1 Evidence ratio for null hypothesis against various alternative hypotheses for the proportion of fish adopting a place strategy in zebrafish species estimated using Bayesian inference. A posteriori probabilities suggest that there is not strong evidence for choosing the null hypothesis over some of the alternative hypotheses. Strong evidence is when the ratio of a null $a$ posteriori probability to an alternative $a$ posteriori probability is less than $1 / 3$ or greater than 3 . For example, if an $a$ priori alternative hypothesis of 0.7 is examined, a value of 1.05 is obtained indicating no strong evidence for choosing this alternative hypothesis over the null, or vice versa.

\section{$\underline{\text { Time Taken on Probe Trial }}$}

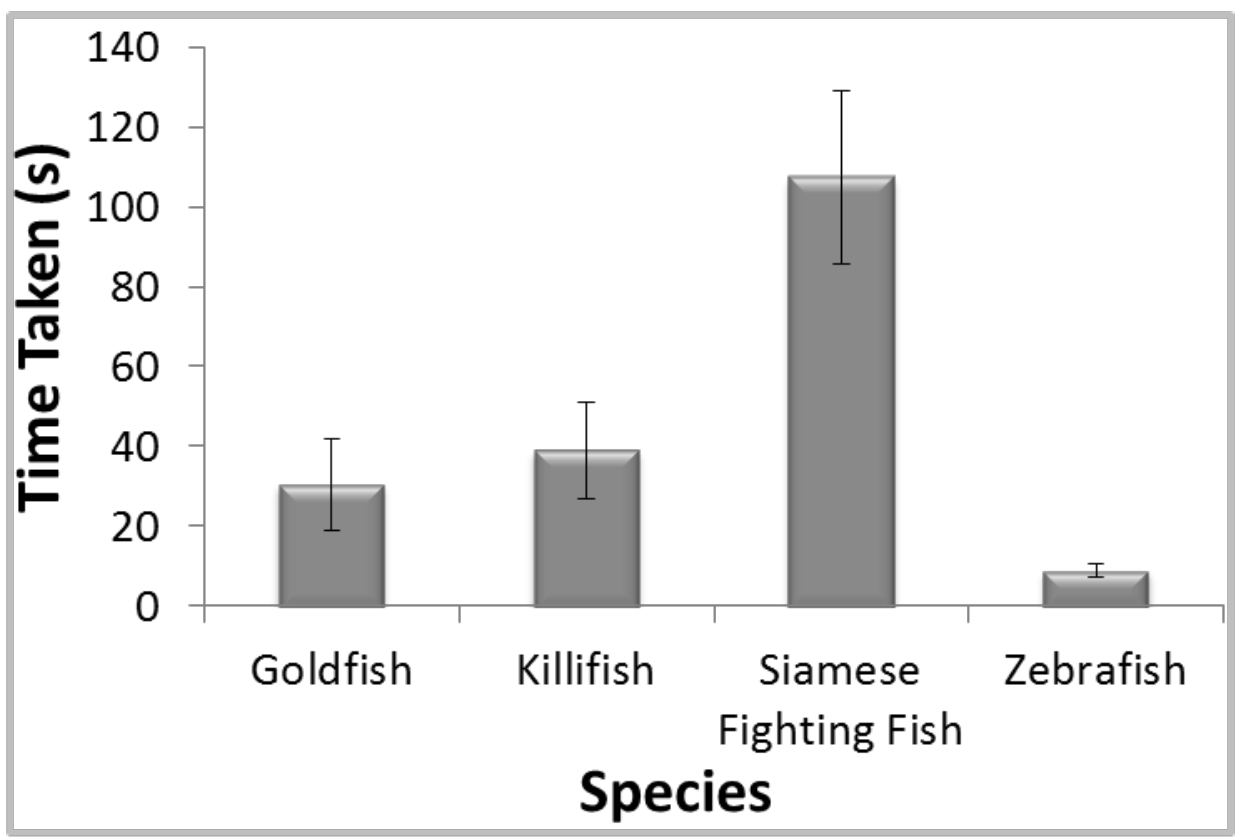

Fig. 6 Bar chart showing the mean time taken in seconds by each species during the probe trial. Error bars indicate the standard error of the mean time taken for individual fish within each species

325 There was a significant main effect of species on time taken during the probe trial: Wald $X^{2}(d f=3)=30.92 ; P<.001$. Posthoc analyses showed Siamese fighting fish took significantly more time (in seconds) than the other three species to complete the probe trial $(P<.001$ in all cases). There were no other

329 significant differences (goldfish vs. killifish; $p=.648$, goldfish vs. zebrafish; $330 P=.250$, killifish vs. zebrafish; $P=.108$ ). (See Fig. 6) 
The aims of this study were to assess the length of time it took four species of fish to learn a spatial memory task and to see what navigational strategy each species preferentially employed in doing so. Results show that zebrafish took significantly more blocks to reach training criterion than the other three species (goldfish, killifish and Siamese fighting fish). Goldfish and killifish also took significantly more blocks than Siamese fighting fish to learn the training task. Such findings correspond with previous studies (Roitblat et al. 1982; Rodriguez et al. 1994) which found that both goldfish and Siamese fighting fish are capable of learning a variety of spatial memory tasks. Although little is

341 known about the spatial memory of Nothobranchius guentheri, the findings in 342 this study do suggest that these fish are also able to learn a spatial memory 343 task and can do so at a faster rate than a more commonly used study species 344 (zebrafish). seconds) to complete an individual trial by the Siamese fighting fish (the

347 largest of the four species and the quickest to learn) was significantly longer in both training $(p<.01)$ and probe trials $(p<.001)$ than the other three species.

349 Similarly, the mean time to complete a training trial by zebrafish (the slowest 350 to learn) was significantly shorter $(p<.001)$ than all the other fish used in these

351 experiments. These findings suggest that taking time to explore the

352 environment may improve the learning process, by allowing the animal to 353 learn from both correct and incorrect trials. In other contexts it has been 354 proposed that animals that are faster explorers and "proactive" might be less 355 accurate whereas animals that are slower explorers and "reactive" might be 356 more accurate in their decision making (Sih et al. 2004). Previous studies 
have also suggested that fish which are slower to explore their environment are more flexible in their behaviour and are quicker to adapt to changes (see: Magnhagen 2012 for a review). However it should be noted that these studies have focused on individual differences within a species and have not compared differences in behavioural flexibility and plasticity across species.

362 We are cautious in our interpretation as another reason for this difference may 363 be simply that Siamese fighting fish are from a different order than the other 364 three species which may have an effect on their adaptation of spatial navigation (Perciformes and Cypriniformes respectively). Further studies manipulating time allowed to complete a trial and its influence on learning 367 speed would also be interesting. Likewise adopting similar analyses used in 368 this study to assess the effects of time taken to make a decision could be applied to explore behavioural outcomes in a variety of species. Although our findings, which show that three species (goldfish,

371 Siamese fighting fish and zebrafish) took significantly less time to complete 372 trials on the last day of training versus the first day of training, would be expected during a learning task, it is unclear why there was no significant

374 difference in the killifish species. This may have been because the killifish 375 were disadvantaged by being the smallest of the four species. However 376 further research is required to fully understand these differences.

377 The findings that three of the four species showed a preference for the 378 adoption of a place strategy suggest that fish, like mammals and birds, are 379 capable of complex spatial memory and that the lack of a hippocampal 380 structure is not necessarily detrimental to the navigational abilities of fish. This 381 has also been argued by others who suggest that the telencephalon in fish, in 
particular the lateral pallium, is homologous to the hippocampus of mammals and birds (Saito \& Watanabe 2006; Spence et al. 2011). Zebrafish did not show a significant preference for the adoption of either a response or a place strategy despite their popularity in previous behavioural and memory studies (Williams et al. 2002; Miklosi \& Andrew 2006; Sison \& Gerlai 2010; Miller \& Gerlai 2012b). However, results from Bayesian inference would suggest an increase in sample size may be required in order to establish whether these fish truly have a preference for either a place or a response strategy. It is possible that the fish were using salient geometric cues external to 391 the maze in order to navigate using what seemed to be a place strategy; this 392 has been suggested to be the case in previous studies using rats as subjects 393 (Ritchie 1947; Cheng 1986). The possible reason why some animals, particularly the zebrafish, did not show a preference for a place strategy may be that they were using geometrical information internal to the maze therefore causing a rotational error on the probe trial and eliciting what seemed to be a response strategy (Ritchie 1947; Cheng 1986). Further experiments could be completed in order to assess the effects of geometrical information on strategy choice.

Another possible reason for the zebrafish species performing differently

401 could be based on zebrafish being a shoaling species which means they are 402 more likely to navigate through their environment in groups (Wright \& Krause 403 2006; Miller \& Gerlai 2012a; Butail et al. 2013). Again, further research is 404 needed to assess whether zebrafish show a difference in navigation or a 405 preference for either strategy when completing the task in shoals. 
Three of the four species were housed individually during

experimentation (killifish, Siamese fighting fish and zebrafish). This isolation could have caused some level of stress, particularly for the shoaling zebrafish species which is highly social. Housing jars were, however, kept beside each other allowing each fish visual contact to conspecifics. Previous studies have suggested that this is satisfactory to reduce isolation stress in such animals and may also induce shoaling movements (e.g. Engeszer et al. 2004; Sison \& Gerlai 2010; Karnik \& Gerlai 2012). In the experimental protocol, fish would complete the trials individually which may have led to isolation stress, particularly in a novel environment. Additionally, it is possible that body size may have had an impact on the results, perhaps placing a disadvantage on the smaller species. There were, however, no observed signs of stress during experimentation with individual fish willingly eating the bloodworm reward on all correct trials, a further indicator that stress levels were minimal during experiments (Carr 2002).

As each fish would receive ten trials per block and multiple fish would be tested on each experimental day, it could be argued that olfactory cues may have been important in the learning of the task and the performance of the probe trial. These effects were controlled for where possible by disturbing the water and any substrate in the tank between each correct trial and by filtering the tank for a minimum of 20 minutes between each fish. Furthermore, a previous study using bloodworm reward and goldfish as a study species suggests that olfaction is not often used by fish during similar maze tests (Saito \& Watanabe 2005). Finally, as place learning was not 
430

431

employed by all fish, it is unlikely that olfactory cues played any significant role on the performance of fish during these experiments.

A previous study suggested that the number of training days has an effect on whether an animal uses a place or response strategy for navigation (Packard \& McGaugh 1996). This study found that rats were more likely to choose a response over a place strategy when they had more training in the experimental set-up. This could explain why the zebrafish, who showed the greatest variation in the number of blocks of trials taken to reach training criteria, showed no significant preference for the adoption of either a place or a response strategy. However, it should be noted that the difference in the set of experiments presented here is that not all fish received the same amount of training. This differed from the previous study (Packard \& McGaugh, 1996) where there was a fixed training schedule before each probe trial. The suggestion that more training could have an impact on navigational strategy also requires further investigation.

It is possible that the fish could have used the wall at the end of one of the goal arms (or the lack of wall at the end of the other) as a navigation beacon. If this was the case, then one would expect the animals to use a simpler US-CS link to learn the task rather than a more complex spatial memory strategy (Karnik \& Gerlai 2012). As global cues external to the maze were not controlled for or analysed during experimentation, it is not possible to conclude what cues the fish used in order to display place learning. Indeed, it has been argued that it is difficult to exclude simpler strategies from any experiment that investigates complex spatial memory processes (Bennett 1996) However, It is surprising that the zebrafish did not show a preference 
455 for the training location if a simple, salient feature was indicating this and

456 could explain performance of the other species. Thus, whether it was

457 beaconing to a cue external to the maze or a set of more complex allocentric

458 features that explain the performance of the other three species, it is clear that

459 the zebrafish were not able to consistently use the same strategy as the other

460 species to learn the location of the food reward. Whilst our experimental setup

461 cannot distinguish between beaconing to a cue and an allocentric strategy, it

462 is worth noting that other experiments, using the same experimental setup

463 (plus maze), have indicated that when the lateral pallium area of the

464 telencephalon is ablated, fish are no longer able to learn place, but retain an

465 ability to use a cue based strategy (Rodriguez et al. 1994; Salas et al. 1996;

466 López et al. 2000; Broglio et al. 2010). Experiments using a controlled salient

467 cue at the rewarded arm would indicate whether zebrafish can learn this task

468 and use it from a novel start point and thus provide further insight into the

469 strategies used in the current experiment.

$470 \quad$ The findings in this study suggest that the spatial memory of fish may

471 be comparable to other animals as three species out of four demonstrated a

472 preference for using a strategy that is often associated with more complex

473 and flexible allocentric navigation when completing the plus maze task .

474 Zebrafish did not demonstrate place learning and showed no significant

475 preference for either a place or response strategy. Further experiments using

476 shoals of zebrafish should be completed to assess whether navigation is

477 facilitated by collective decision-making in this species (Couzin et al. 2005).

478 Regardless of strategy choice, these findings suggest that all four species are

479 able to learn a spatial memory task, reinforcing previous findings that fish are 
a useful animal model for the study of cognitive behavioural tasks (Rodriguez

481 et al. 1994; Braithwaite \& De Perera 2006; White \& Brown 2014).

482

483

484

485

486

487

488

489

490

491

492

493

\section{Acknowledgements}

The authors would like to acknowledge the following individuals for assistance with lab. set-up and fish husbandry: Georgina Glaser, Sinéad Smith, Chris Preshaw, Gillian Riddell and other members of the technical staff at the School of Biological Sciences, QUB. We would also like to thank the funding bodies of this research: The Department of Employment and Learning, Northern Ireland and Queen's University Belfast. Finally, we would like to thank Ken Cheng and Hannah White for advice on statistics.

\section{Conflict of Interest}

The authors declare that they have no conflict of interest.

\section{References}

Andrews, C., 2002. An Interpet Guide to Fancy Goldfish, Surrey: Interpet Publishing.

Bennett, A.T., 1996. Do animals have cognitive maps? J Exp Biol, 199, pp.219-224.

Braithwaite, V.A. \& De Perera, T.B., 2006. Short-range orientation in fish: how fish map space. Mar Freshw Behav Phy, 39(1), pp.37-47.

Broglio, C. et al., 2010. Selective involvement of the goldfish lateral pallium in spatial memory. Behav Brain Res, 210(2), pp.191-201.

Broglio, C., Rodriguez, F. \& Salas, C., 2003. Spatial cognition and its neural basis in teleost fishes. Fish Fish, 4, pp.247-255.

Brown, C., 2014. Fish intelligence, sentience and ethics. Anim Cogn, 18, pp.1-17.

Butail, S., Bartolini, T. \& Porfiri, M., 2013. Collective response of zebrafish shoals to a free-swimming robotic fish. PloS One, 8(10), p.e76123.

Carr, J.A., 2002. Stress, neuropeptides, and feeding behavior: a comparative perspective. Integr Comp Bio, 42(3), pp.582-590. 
Casebolt, D., Speare, D. \& Horney, B., 1998. Care and use of fish as laboratory animals: Current state of knowledge. Lab Anim Sci, 48(2), pp.124-135.

Cheeseman, J.F. et al., 2014. Way-finding in displaced clock-shifted bees proves bees use a cognitive map. P Natl A Sci USA, 111(24), pp.894954.

Cheng, K., 1986. A purely geometric module in the rat's spatial representation. Cognition, 23, pp. 149-178.

Clayton, N.S. \& Krebs, J.R., 1995. Memory in food-storing birds: from behaviour to brain. Curr Opin Neurobiol, 5(2), pp.149-154.

Couzin, I.D. et al., 2005. Effective leadership and decision-making in animal groups on the move. Nature, 433(7025), pp.513-6.

Dittman, A.H. \& Quinn, T.P., 1996. Homing in pacific salmon: mechanisms and ecological basis. J Exp Biol, 199, pp.83-91.

Engeszer, R.E., Ryan, M.J. \& Parichy, D.M., 2004. Learned social preference in zebrafish. Curr Biol, 14(10), pp.881-4.

Genade, T. et al., 2005. Annual fishes of the genus Nothobranchius as a model system for aging research. Aging Cell, 4(5), pp.223-233.

Van Gerven, D.J.H. et al., 2012. Direct measurement of spontaneous strategy selection in a virtual Morris water maze shows females choose an allocentric strategy at least as often as males do. Behav Neurosci, 126(3), pp.465-78.

Gordon, M. \& Axelrod, H., 1968. Siamese Fighting Fish, New Jersey: T.F.H Publications, Inc.

Guidelines for the Use of Animals (2012) Guidelines for the treatment of animals in behavioural research and teaching. Anim Behav, 83, 301-309

Hamilton, D.A. et al., 2009. Control of rodent and human spatial navigation by room and apparatus cues. Behav Process, 81(2), pp.154-69.

Herrera, M. \& Jagadeeswaran, P., 2004. Annual fish as a genetic model for aging. J Gerontol A Biol Sci Med Sci, 59(2), pp.101-7.

Iaria, G. et al., 2009. Age differences in the formation and use of cognitive maps. Behav Brain Res, 196(2), pp.187-91.

Karnik, I. \& Gerlai, R., 2012. Can zebrafish learn spatial tasks? An empirical analysis of place and single CS-US associative learning. Behav Brain Res, 233(2), pp.415-421. 
Kishi, S., 2004. Functional Aging and Gradual Senescence in Zebrafish. Ann NY Acad Sci, 1019, pp.521-526.

Lamb, E.A., Echevarria, D.J. \& Jouandot, D.J., 2012. The utility of the T-maze in assessing learning, memory, and models of neurological disorders in the zebrafish. Behaviour, 149(10-12), pp.1081-1097.

López, J.C. et al., 2000. Dissociation of place and cue learning by telencephalic ablation in goldfish. Behav Neurosci, 114(4), pp.687-699.

Magnhagen, C., 2012. Personalities in a crowd: What shapes the behaviour of Eurasian perch and other shoaling fishes? Curr Zoo, 58(1), pp.35-44.

Maguire, E. et al., 2000. Navigation-related structural change in the hippocampi of taxi drivers. PNAS, 97(8), pp.4398-4403.

Miklósi, a \& Andrew, R.J., 1999. Right eye use associated with decision to bite in zebrafish. Behav Brain Res, 105(2), pp.199-205.

Miklosi, A. \& Andrew, R., 2006. The zebrafish as a model for behavioral studies. Zebrafish, 3(2), pp.227-234.

Miller, N. \& Gerlai, R., 2012a. From schooling to shoaling: patterns of collective motion in zebrafish (Danio rerio). PloS one, 7(11), p.e48865.

Miller, N. \& Gerlai, R., 2012b. Automated tracking of zebrafish shoals and the analysis of shoaling behaviour. In: Kalueff, A.V. \& Steward, A.M. Zebrafish Protocols for Neurobehavioral Research, pp. 217-230. New Jersey: Humana Press.

O'Keefe, J. \& Nadel, L., 1978. The Hippocampus as a Cognitive Map, Oxford: Clarendon Press.

Odling-Smee, L. \& Braithwaite, V.A., 2003. The influence of habitat stability on landmark use during spatial learning in the three-spined stickleback. Anim Behav, 65(4), pp.701-707.

Packard, M.G. \& McGaugh, J.L., 1996. Inactivation of hippocampus or caudate nucleus with lidocaine differentially affects expression of place and response learning. Neurobiol Learn Mem, 65(1), pp.65-72.

Pike, T.W. et al., 2010. Learning by proportional observation in a species of fish. Behav Ecol, 21(3), pp.570-575.

Portavella, M. \& Vargas, J., 2005. Emotional and spatial learning in goldfish is dependent on different telencephalic pallial systems. Eur J Neurosci, 21, pp.2800-2806. 
Pravosudov, V. V. \& Roth II, T.C., 2013. Cognitive ecology of food hoarding: The evolution of spatial memory and the Hippocampus. Annu Rev Ecol Evol S, 44(1), pp.173-193.

Ritchie, B.F., 1947. Studies in spatial learning III: Two paths to the same location and two paths to two different locations. J Expl Psychol, 37(1), pp. 25-38.

Rodriguez, F. et al., 1994. Performance of goldfish trained in allocentric and egocentric maze procedures suggests the presence of a cognitive mapping system in fishes. Anim Learn Behav, 22(4), pp.409-420.

Roitblat, H., Tham, W. \& Golub, L., 1982. Performance of Betta splendens in a radial arm maze. Anim Learn Behav, 10(1), pp.108-114.

Saito, K. \& Watanabe, S., 2006. Deficits in acquisition of spatial learning after dorsomedial telencephalon lesions in goldfish. Behav Brain Res, 172(2), pp.187-94.

Saito, K. \& Watanabe, S., 2005. Experimental analysis of spatial learning in goldfish. Psychol Rec, 55, pp.647-662.

Salas, C. et al., 1996. Spatial Learning and Memory Deficits After Telencephalic Ablation in Goldfish Trained in Place and Turn Maze Procedures. Behav Neurosci, 110(5), pp.965-980.

Shapiro, M.S. \& Jensen, A.L., 2009. Parameters of rewards on choice behavior in Siamese fighting fish (Betta splendens). Behav Process, 82(1), pp.30-8.

Shelton, A.L. \& McNamara, T.P., 2001. Systems of spatial reference in human memory. Cognitive Psychol, 43(4), pp.274-310.

Shettleworth, S.J. \& Westwood, R.P., 2002. Divided attention, memory, and spatial discrimination in food-storing and nonstoring birds, black-capped chickadees (Poecile atricapilla) and dark-eyed Juncos (Junco hyemalis). J Exp Psychol Anim B, 28(3), pp.227-241.

Sih, A., Bell, A. \& Johnson, J.C., 2004. Behavioral syndromes: An ecological and evolutionary overview. Trends Ecol Evol, 19(7), pp.372-378.

Sison, M. \& Gerlai, R., 2010. Associative learning in zebrafish (Danio rerio) in the plus maze. Behav Brain Res, 207(1), pp.99-104.

Spence, R., Magurran, A.E. \& Smith, C., 2011. Spatial cognition in zebrafish: the role of strain and rearing environment. Anim Cogn, 14(4), pp.607-12.

Thomas, P.O.R. et al., 2008. Does defection during predator inspection affect social structure in wild shoals of guppies? Anim Behav, 75(1), pp.43-53. 

learning versus response learning. J Exp Psychol, 36(3), pp.221-229.

Tolman, E.C., 1948. Cognitive maps in rats and men (1). Psychol Rev, 55(4), pp.189-208.

Tropepe, V. \& HL Sive, 2003. Can zebrafish be used as a model to study the neurodevelopmental causes of autism? Genes Brain Behav, 2, pp.268281.

White, G.E. \& Brown, C., 2014. A comparison of spatial learning and memory capabilities in intertidal gobies. Behav Ecol Sociobiol, 68(9), pp.13931401.

Williams, F.E., White, D. \& Messer, W.S., 2002. A simple spatial alternation task for assessing memory function in zebrafish. Behav Process, 58(3), pp.125-132.

Wolbers, T. \& Hegarty, M., 2010. What determines our navigational abilities? Trends Cogn Sci, 14(3), pp.138-46.

Wright, D. \& Krause, J., 2006. Repeated measures of shoaling tendency in zebrafish (Danio rerio) and other small teleost fishes. Nat Protoc, 1(4), pp.1828-31.

\section{Figure Captions}

Fig. 1 Layout of experimental T-maze formed by blocking arm directly opposite start arm with a piece of Perspex. Reverse layout would be used as the T-maze for probe trials

Fig. 2 Boxplot showing task acquisition time for each species. Rectangular boxes display 25th \& 75th quartiles and the median. Whiskers display 90th percentile of the data with outliers outside this range marked with an $\mathrm{X}$ on the plot. The dotted line displays the minimum number of blocks to reach training criteria within each species (3 blocks in all species). On the graphical display, asterisks are used to show significant differences between species (a single asterisk (*) indicates $P \leq .05$ and double asterisks $\left(^{* *}\right)$ indicate $P<.001$ )

Fig. 3 Bar chart showing the mean time taken in seconds by each species to complete the training trials. Error bars indicate the standard error of the mean time taken for individual fish within each species

Fig. 4 Bar chart showing mean time taken in seconds by each species to complete trials on the first day of training versus on the last day of training. Error bars indicate the standard error of the mean time taken for individual fish within each species

Fig. 5 Bar chart showing the number of fish in each species that adopted either a place or a response strategy during probe trials. An asterisk (*) indicates that there was a significant difference in the number of fish that adopted a place strategy over a response strategy $(P<0.05)$ $(n=20$ in all species)

Fig. 6 Bar chart showing the mean time taken in seconds by each species during the probe trial. Error bars indicate the standard error of the mean time taken for individual fish within each species proportion of fish adopting a place strategy in zebrafish species estimated using Bayesian inference. A posteriori probabilities suggest that there is not strong evidence for choosing the null hypothesis over some of the alternative hypotheses. Strong evidence is when the ratio of a 
658

null a posteriori probability to an alternative a posteriori probability is less than $1 / 3$ or greater

659 than 3. For example, if an a priori alternative hypothesis of 0.7 is examined, a value of 1.05 is

660 obtained indicating no strong evidence for choosing this alternative hypothesis over the null, or vice versa.

662 